Schwere systemische Reaktionen wurden nicht berichtet. Über oralen Pruritus, Mundödeme oder Kratzen im Hals berichteten $20 \%$ (12 SQ-HDM) respektive $13 \%$ (6 SQ-HDM) in den SLIT-Gruppen und $3 \%$ in der Placebogruppe.

Fazit: Bei Asthmatikern mit Hausstaubmilbenallergie, deren Beschwerden nur unzureichend mit ICS kontrollierbar sind, lässt sich während einer ICS-Reduktion mithilfe einer SLIT einer moderatenoderschwerenAsthmaexazerbation vorbeugen, so die Autoren. Das absolute Risiko sinke ungefähr um 9-10\%, wobei der Effekt überwiegend der Prävention moderater Exazerbationen geschuldet sei, wie die Studienautoren erklären. Als nächsten Schritt fordern sie, die Wirksamkeit und Sicherheit der SLIT über einen längeren Zeitraum hin zu überprüfen.

Dr. Dagmar Kraus

Virchow JC et al. Efficacy of a House Dust Mite Sublingual Allergen Immunotherapy Tablet in Adults With Allergic Asthma. JAMA 2016;315: 1715-25

\section{Schützen Mikroorganismen im Darm vor Allergien?}

\author{
Als Ursache für die deutliche Zunahme allergischer Erkrankungen in den \\ letzten Jahrzehnten spielen möglicherweise Veränderungen im intestinalen \\ Mikrobiom eine entscheidende Rolle.
}

$B^{\mathrm{e}}$ eginnend mit dem Geburtsvorgang wird die Darmflora des Menschen sukzessive mit tausenden verschiedener Bakterienspezies besiedelt. Je höher die mikrobielle Vielfalt, so legen die Ergebnisse zahlreicher Studien nahe, desto geringer ist offenbar das Risiko für allergische Erkrankungen. Insbesondere die ersten Monate nach der Geburt scheinen ein kritisches Zeitfenster für die Entwicklung des Mikrobioms zu sein, betont Caspar Ohnmacht vom Zentrum Allergie \& Umwelt (ZAUM) an der Technischen Universität und am Helmholtz Zentrum München. Eine antibiotische Behandlung in dieser Zeitspanne könne die Zusammensetzung der Darmbakterien langfristig aus dem Gleichgewicht bringen.

Tatsächlich wurde in Studien nachgewiesen, dass antibiotisch behandelte Kinder ein erhöhtes Risiko haben, Allergien zu entwickeln. Auch der seit Mitte des letzten Jahrhunderts deutlich erhöhte Hygienestandard, der sinkende Anteil an Kindern, die mit Tieren aufwachsen, sowie die steigende Rate der Geburten per Kaiserschnitt scheinen dazu beigetragen zu haben, dass atopische Erkrankungen in den letzten Jahrzehnten zugenommen haben.

Über welche Mechanismen diese Veränderungen stattgefunden haben, ist bislang nicht genau geklärt. Nach Ohnmacht hat sich das Immunsystem des Menschen parallel $\mathrm{zu}$ den mit ihm in Symbiose lebenden Mikroorganismen entwickelt. Infolgedessen sei eine Vielzahl von Mechanismen entstanden, die diese beiden Systeme im Gleichgewicht halten. Ein essenzieller Bestandteil dieser Regulation sind Tregs, regulatorische TZellen, die im Darm in großer Zahl und mit einiger Variabilität exprimiert sind.

Während man bisher der Ansicht war, dass Bakterien eine Typ-1-Immunantwort induzieren und damit einer proallergischen Typ-2-Immunantwort entgegenwirken, haben neuere Studien Hinweise darauf erbracht, dass diese Rolle auch bestimmte Tregs übernehmen können, die sich nicht wie üblich im Thymus, sondern erst im Darmgewebe aus naiven T-Helferzellen differenziert haben (peripher induzierte Foxp3-exprimierende Tregs).

Laut Ohnmacht begünstigen Tregs die Bildung, Diversifikation und Selektion von IgA, welches nach Transport ins Darmlumen an die dortigen Bakterien bindet. Somit tragen die Tregs zur Aufrechterhaltung der Toleranz unseres Immunsystems gegenüber der Darmflora und damit zur bakteriellen Vielfalt im Darm bei. Darüber hinaus sind die Immunregulatoren wahrscheinlich auch für die Toleranz gegenüber harmlosen Fremdantigenen verantwortlich.

In Studien mit Mäusen hat man herausgefunden, dass bei Tieren mit einem Defekt in der Treg-Differenzierung die IgE-Spiegel steigen; zusätzlich fand sich auch eine erhöhte Anzahl an
Typ-2-Helferzellen in den Schleimhäuten.

Positiv auf die Induktion von Tregs wirken sich offenbar kurzkettige Fettsäuren (SCFA) aus. Diese entstehen, wenn komplexe Kohlenhydrate durch Mikroorganismen fermentiert werden. Interessanterweise konnte man zeigen, dass Kinder mit atopischen Erkrankungen geringere SCFA-Spiegel haben als gesunde Kinder.

Derzeit lassen sich diese Erkenntnisse noch nicht therapeutisch nutzen. In Tierversuchen schützte allerdings die Supplementierung mit SCFA vor allergischem Asthma. Ein weiterer Ansatzpunkt für die Therapieforschung ergibt sich aus der Tatsache, dass offenbar auch bestimmte Clostridienspezies die Induktion von Tregs bewirken können. Und auch einzelne bakterielle Bestandteile wie das von Bacteroides fragilis gebildete Polysaccharid A fördern möglicherweise die Bildung der Immunregulatoren. Dr. Elke Oberhofer

Ohnmacht C. Microbiota, regulatory T cell subsets, and allergic disorders. Allergo J Int 2016; 25; 114-7

\section{Kommentar}

Eine ausgezeichnete sehr aktuelle Übersichtsarbeit, deren Lektüre sich auch im Original lohnt, besonders für diejenigen unter unseren Lesern, die sich für Mechanismen der Immunregulation und dem Zusammenspiel zwischen Darmmikrobiom und Immunantwort interessieren.

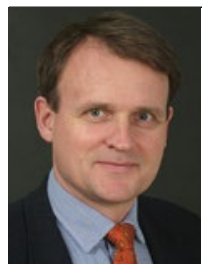

Prof. Dr. Thilo Jakob Direktor der Klinik für Dermatologie und Allergologie Universitätsklinikum Gießen u. Marburg, Standort Gießen 